\title{
Complete recovery from paraneoplastic anti-NMDAR encephalitis associated with a small ovarian teratoma following a laparoscopic salpingo-oophorectomy: A case report
}

\author{
KENJI IMAI, TAKESHI FUKUDA, TAKUMA WADA, MASARU KAWANISHI, MAKOTO YAMAUCHI, \\ YASUNORI HASHIGUCHI, TOMOYUKI ICHIMURA, TOMOYO YASUI and TOSHIYUKI SUMI
}

Department of Obstetrics and Gynecology, Graduate School of Medicine, Osaka City University, Osaka 545-8585, Japan

Received July 3, 2014; Accepted February 19, 2015

DOI: $10.3892 / \mathrm{etm} .2015 .2344$

\begin{abstract}
Anti-N-methyl-D-aspartate receptor (anti-NMDAR) encephalitis is a severe but treatment-responsive disorder, initially identified in young women with an ovarian teratoma. Symptoms include mood, behavior and personality irregularities that resemble acute psychosis. The present study reports the rare case of a patient with anti-NMDAR encephalitis and severe neurological symptoms, that exhibited a rapid recovery following a laparoscopic salpingo-oophorectomy. The 39-year-old woman was admitted to the Department of Obstetrics and Gynecology, Osaka City University Graduate School of Medicine (Osaka, Japan) with a 5-day history of fever and stomach ache. One week later, the patient developed hallucinations and emotional lability. Initially, a diagnosis of limbic or herpes encephalitis was considered; thus, the patient was administered acyclovir and received steroid pulse therapy. However, the patient subsequently developed apnea, and in response, a tracheal intubation, mechanical ventilation and plasmapheresis were performed. Anti-NMDAR encephalitis was subsequently considered as a diagnosis and mediastinal and pelvic computed tomography (CT) examinations were conducted to detect for the presence of a teratoma. A $24 \times 24-\mathrm{mm}$ cystic lesion was identified in the pelvis from an abdominal CT scan and the lesion appeared to be an ovarian teratoma. In addition, serum and cerebrospinal fluid samples were collected, and were found to test positive for anti-NMDAR antibodies. A laparoscopic salpingo-oophorectomy was performed, which resulted in rapid improvement of the patient's mental symptoms, followed by a complete recovery.
\end{abstract}

Correspondence to: Dr Takeshi Fukuda, Department of Obstetrics and Gynecology, Graduate School of Medicine, Osaka City University, 1-4-3 Asahimachi, Abeno-ku, Osaka 545-8585, Japan E-mail: takeshif@med.osaka-cu.ac.jp

Key words: anti-N-methyl-D-aspartate receptor encephalitis, ovarian teratoma, laparoscopic salpingo-oophorectomy

\section{Introduction}

Ovarian teratoma-associated encephalitis was first reported in 1997 (1,2). In 2007, an association was identified between this type of encephalitis and N-methyl-D-aspartate receptor (NMDAR) antibodies (3). NMDAR is a ligand-gated cation channel, comprising NR1 and NR2 subunits, that serves crucial functions in synaptic transmission and plasticity (4). Overactivation of NMDAR may result in excitotoxicity, which contributes to the development of epilepsy, dementia and stroke. Conversely, low NMDAR activity results in symptoms that resemble schizophrenia (5). The autoantibodies produced in patients with concurrent anti-NMDAR encephalitis and teratoma exhibit cross-reactivity with the NMDAR in the teratoma, binding to the NR subunits of NMDAR following entry into the cerebrospinal fluid.

Anti-NMDAR encephalitis is a severe condition that is treatment-responsive. The disorder was initially identified in young females with an ovarian teratoma. These patients developed mood, behavior and personality abnormalities that resembled acute psychosis. The symptoms of anti-NMDAR encephalitis include neuropsychiatric symptoms, hyperkinesia, autonomic dysfunction, hypoventilation and epilepsy. Anti-NMDAR encephalitis is generally considered to be a paraneoplastic disease due to the rapid clinical improvement typically observed in patients following an ovariectomy. However, in certain patients, no teratoma or other tumor types are detected; thus, the exact prevalence and incidence of this disease remain unknown (2).

The present study reports a rare case involving a patient with anti-NMDAR encephalitis and severe neurological symptoms. Following a laparoscopic salpingo-oophorectomy, the patient exhibited a rapid recovery.

\section{Case report}

Written informed consent was obtained from the patient's family. A 39-year-old woman with no significant medical history was admitted to the the Department of Obstetrics and Gynecology, Osaka City University Graduate School of Medicine (Osaka, Japan) with a 5-day history of fever and stomach ache. The patient's symptoms initially resembled 


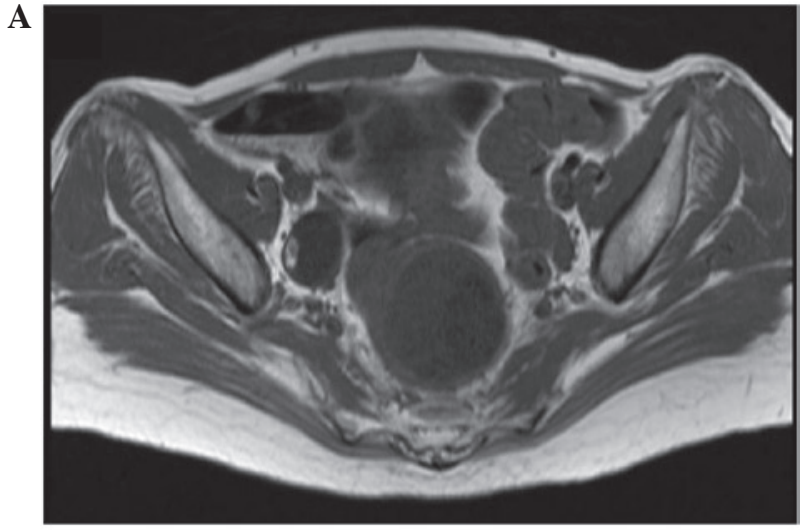

B

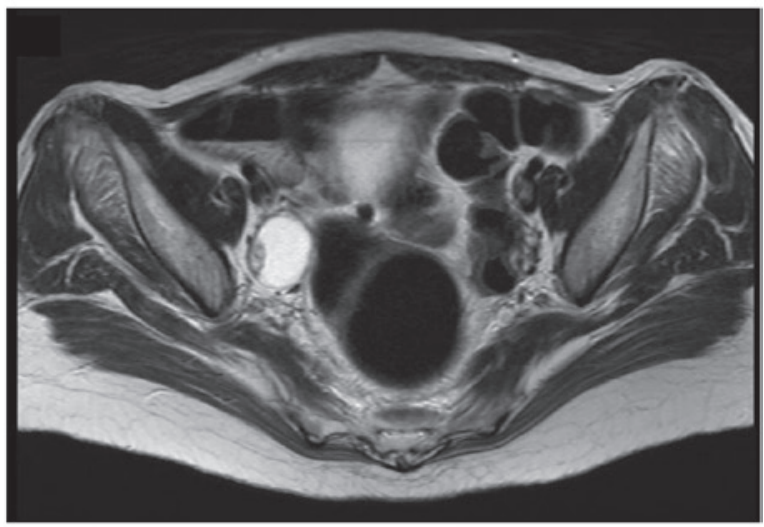

C

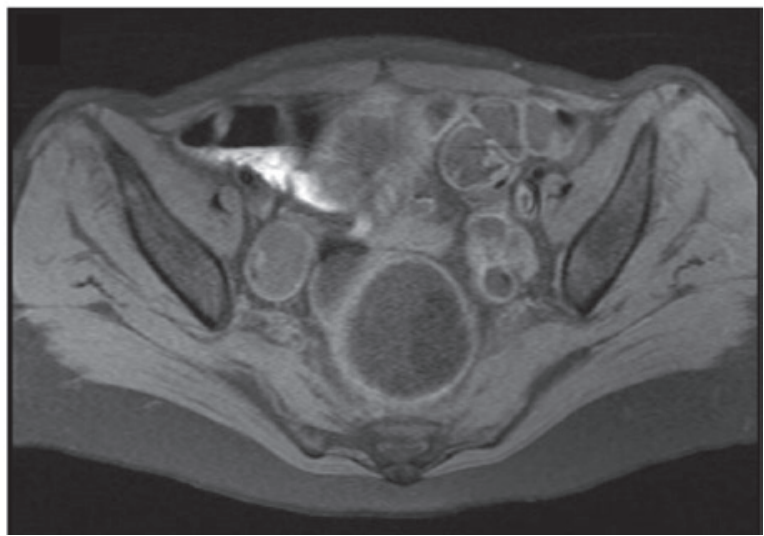

Figure 1. Magnetic resonance imaging (MRI) examinations. (A) T1-weighted MRI scan shows a low-intensity mass $(30 \times 23 \mathrm{~mm})$ containing a small high-intensity region in the right ovary. (B) T2-weighted MRI scan reveals a high-intensity mass $(30 \times 23 \mathrm{~mm}$ ) in the right ovary. (C) T1-weighted fat-suppressed MRI scan shows suppressed intensity in the high-intensity regions of the T1- and T2-weighted images.

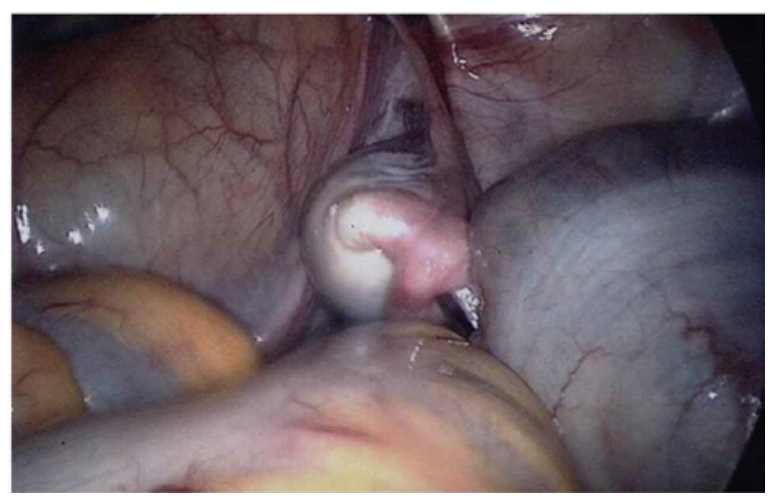

Figure 2. Intraoperative photograph of the mass in the right-hand side of the uterus $(\sim 3 \mathrm{~cm})$.

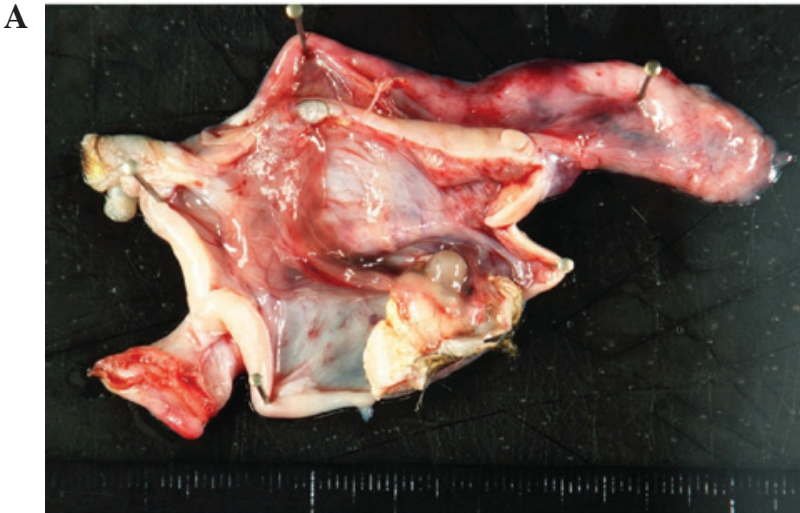

B

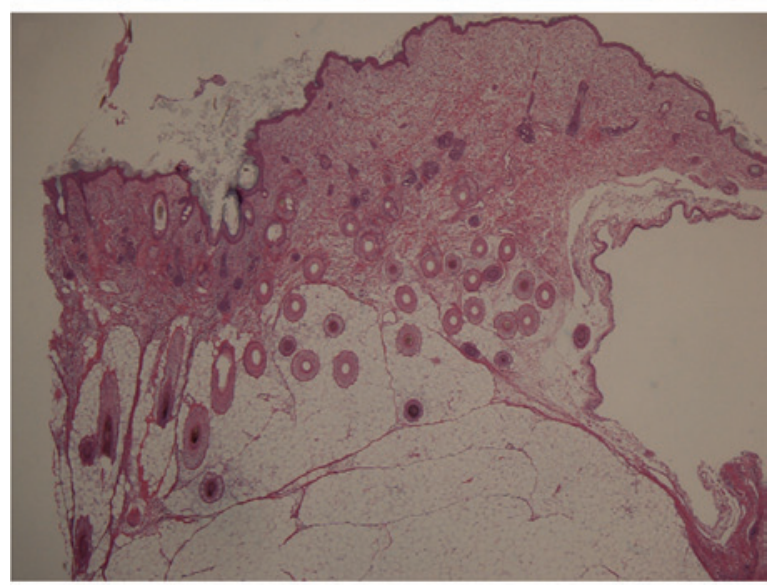

C

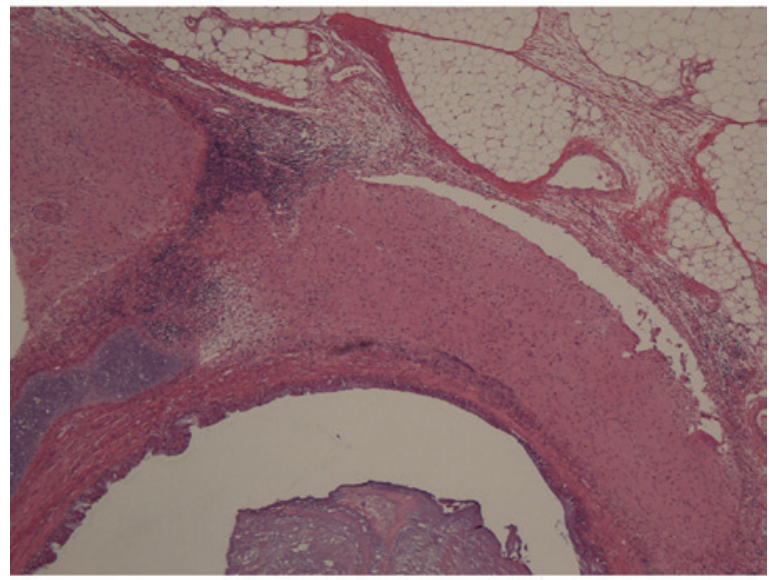

D

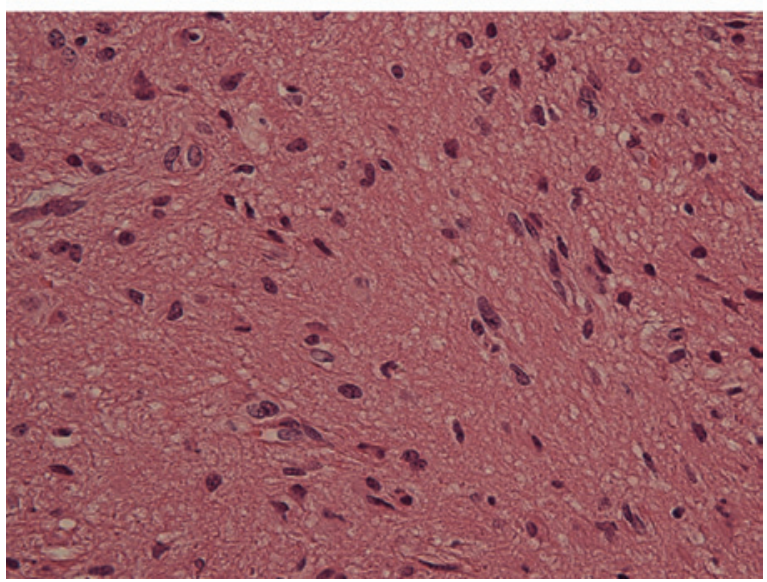

Figure 3. Macroscopic and microscopic images of the extracted mass. (A) Macroscopic image of the extracted tumor, which contains adipose tissue and hair. (B) Microscopic image (magnification, x4). (C) Microscopic image showing the presence of mature skin with sebaceous glands and hair follicles (magnification, x20). (D) Microscopic image showing the high level of differentiation in the central nervous tissue (magnification, $\mathrm{x} 400$ ). 
those of the common cold. However, one week later, the patient experienced hallucinations and emotional lability. Blood testing and brain computed tomography (CT) scans indicated no abnormalities. However, the condition progressed, and the patient exhibited deteriorated emotional lability, increased disorientation and progressive dyskinesia, which was characterized by chorea of the body and limbs. An examination of the cerebrospinal fluid detected a cell count of $77-\mathrm{mm}^{3}$, with a high number of monocytes. However, the head CT examination performed upon arrival revealed no notable abnormalities. Initially, limbic-herpes encephalitis was considered as a diagnosis; thus, the patient was administered acyclovir (1,5000 mg-day, 7 days) and received steroid pulse therapy (solu-medrol 1,000 mg-day, 3 days, 3 courses). However, the patient subsequently developed apnea. Therefore, a tracheal intubation, mechanical ventilation and plasmapheresis were performed. A lumbar puncture, serum viral analysis, head CT and magnetic resonance imaging (MRI) examinations were unable to clarify the etiology of the patient's symptoms. Therefore, anti-NMDAR encephalitis was considered as a potential diagnosis, and mediastinal and pelvic CT examinations were conducted to detect the presence of a teratoma. An abdominal CT scan revealed a $24 \times 24-m m$ cystic lesion in the pelvis, which appeared to be an ovarian teratoma. Furthermore, pelvic MRI revealed a right ovarian teratoma (Fig. 1). In addition, serum and cerebrospinal fluid samples were collected, and were determined to be positive for anti-NMDAR antibodies. A laparoscopic right salpingo-oophorectomy was subsequently performed. Intraoperatively, the right ovary was observed to be enlarged to $30 \mathrm{~mm}$, while the left ovary remained at a normal size (Fig. 2). Pathological examination confirmed the diagnosis of a mature right ovarian cystic teratoma, containing neural tissue (Fig. 3).

The patient underwent postoperative steroid therapy (prednisolone $20 \mathrm{mg}$-day) until discharge, and the condition was observed to improve gradually. Mechanical respiratory support was no longer required by postoperative day 15 , and the patient opened her eyes and began speaking on postoperative day 20, until which the patient had been comatose. Furthermore, the patient's mental symptoms exhibited notable improvement, and the patient achieved complete recovery by postoperative day 53 .

\section{Discussion}

Anti-NMDAR encephalitis is an autoimmune antibody-mediated form of limbic encephalitis, that was initially reported by Dalmau et al in 2007 (3). Anti-NMDAR encephalitis frequently occurs in young women, at a median age of 23 years (5). Commonly observed symptoms include psychiatric symptoms, involuntary movement of the face and limbs, consciousness disturbances and central hypoventilation. The clinical progression of anti-NMDAR encephalitis typically begins with symptoms that resemble the common cold, which subsequently progress to a period of psychiatric symptoms, followed by an immobile period and a hyperactive period (6). This clinical course was observed in the present case. A definitive diagnosis can be achieved following the detection of anti-NMDAR antibodies in the cerebrospinal fluid and serum.
A previous study reported that $40 \%$ of patients with anti-NMDAR encephalitis are aged $<18$ years (median age, 14 years), while older female patients develop ovarian teratomas more frequently compared with younger patients. Statistically, $31 \%$ of female patients aged $<18$ years and $56 \%$ of female patients aged $>18$ years exhibited teratomas (7). However, the incidence of paraneoplastic anti-NMDAR encephalitis remains unknown.

A tumorectomy is an effective treatment method when cases of teratoma are detected at an early stage (5). In addition, steroid pulse therapy, gamma globulin therapy, plasmapheresis and immunosuppressant therapy are effective treatment methods $(8,9)$. Although $75 \%$ of patients recover completely, severe and prolonged disease is possible, and the mortality rate of anti-NMDAR encephalitis is 7\% (5).

The present study described a rare case involving a patient with anti-NMDAR encephalitis who rapidly recovered from severe neurological symptoms following a laparoscopic salpingo-oophorectomy. The patient exhibited neuropsychiatric symptoms, hyperkinesia, autonomic dysfunction, hypoventilation and epilepsy; however, no signs of meningitis were observed. The etiology of the patient's symptoms was unable to be clarified by a lumbar puncture, serum viral analysis, head CT or MRI examination. Therefore, anti-NMDAR encephalitis was considered as a potential diagnosis. Abdominal CT examination revealed a teratoma in the patient's right ovary. Furthermore, anti-NMDAR antibodies were detected in the cerebrospinal fluid and serum of the patient. Thus, the diagnosis of anti-NMDAR encephalitis was confirmed.

In conclusion, gynecologists should be aware that small ovarian teratomas may induce anti-NMDAR encephalitis. Removal of the NMDAR-expressing tumor reduces the serum and cerebrospinal fluid levels of the pathological autoantibody (10). The present case report demonstrates that complete recovery from the severe neurological symptoms of anti-NMDAR encephalitis is possible following surgical resection.

\section{References}

1. Nokura K, Yamamoto H, Okawara Y, Koga H, Osawa H and Sakai K: Reversible limbic encephalitis caused by ovarian teratoma. Acta Neurol Scand 95: 367-373, 1997.

2. Okamura H, Oomori N and Uchitomi Y: An acutely confused 15-year-old girl. Lancet 350: 488, 1997.

3. Dalmau J, Tüzün E, Wu HY, Masjuan J, Rossi JE, Voloschin A, Baehring JM, Shimazaki H, Koide R, King D, et al: Paraneoplastic anti-N-methyl-d-aspartate receptor encephalitis associated with ovarian teratoma. Ann Neurol 61: 25-36, 2007.

4. Lynch DR, Anegawa NJ, Verdoorn T and Pritchett DB: N-methyl-D-aspartate receptors: different subunit requirements for binding of glutamate antagonists, glycine antagonists and channel-blocking agents. Mol Pharmacol 45: 540-545, 1994.

5. Dalmau J, Gleichman AJ, Hughes EG, Rossi JE, Peng X, Lai M, Dessain SK, Rosenfeld MR, Balice-Gordon R and Lynch DR: Anti-NMDA-receptor encephalitis: Cases series and analysis of the effect of antibodies. Lancet Neurol 7: 1091-1098, 2008.

6. Iizuka T, Sakai F, Ide T, Monzen T, Yoshii S, Iigaya M, Suzuki K, Lynch DR, Suzuki N, Hata T and Dalmau J: Anti-NMDA receptor encephalitis in Japan: Long-term outcome without tumor removal. Neurology 70: 504-511, 2008.

7. Florance NR, Davis RL, Lam C, Szperka C, Zhou L, Ahmad S, Campen CJ, Moss H, Peter N, Gleichman AJ, et al: Anti-N-methyl-D-aspartate receptor (NMDAR) encephalitis in children and adolescents. Ann Neurol 66: 11-18, 2009. 
8. Ishiura H, Matsuda S, Higashihara M, Hasegawa M, Hida A, Hanajima R, Yamamoto T, Shimizu J, Dalmau J and Tsuji S: Response of anti-NMDA receptor encephalitis without tumor to immunotherapy including rituximab. Neurology 71: 1921-1923, 2008

9. Sansing LH, Tüzün E, Ko MW, Baccon J, Lynch DR and Dalmau J: A patient with encephalitis associated with NMDA receptor antibodies. Nat Clin Pract Neurol 3: 291-296, 2007.
10. Seki M, Suzuki S, Iizuka T, Shimizu T, Nihei Y, Suzuki N and Dalmau J: Neurological response to early removal of ovarian teratoma in anti-NMDAR encephalitis. J Neurol Neurosurg Psychiatry 79: 324-326, 2008. 